\title{
Effects of restraint stress and high-fat diet on osseointegration of titanium implants: an experimental study
}

\author{
Serkan DUNDAR ${ }^{(a)}$ \\ Alihan BOZOGLAN(a) iD \\ Ozgur BULMUS(b) iD \\ Samet TEKIN(c) iD \\ Tuba Talo YILDIRIM(a) iD \\ Mustafa KIRTAY(d) \\ Vesile Elif TOY(e) ID \\ Mehmet GUL (f) \\ Merve Yilmaz BOZOGLAN ${ }^{(g)}$ \\ (a) Firat University, Faculty of Dentistry, \\ Department of Periodontology, Elazig, Turkey. \\ (b)Firat University, Faculty of Medicine, \\ Department of Physiology, Elazig, Turkey. \\ (c)Firat University, Faculty of Dentistry, \\ Department of Prosthodontics, Elazig, Turkey. \\ (d) Private Practice, Toronto, Canada. \\ (e)|nonu University, Faculty of Dentistry, \\ Department of Periodontology, Malatya, Turkey. \\ (f) Harran University, Faculty of Dentistry, \\ Department of Periodontology, Sanliurfa, Turkey \\ (g)Ministry of Health, Elazig Health Directorate, \\ Department of Paharmacology, Elazig, Turkey
}

Declaration of Interests: The authors certify that they have no commercial or associative interest that represents a conflict of interest in connection with the manuscript.

\section{Corresponding Author:}

Serkan Dundar

E-mail: sdundar@firat.edu.tr,

dtserkandundar@gmail.com

https://doi.org/10.1590/1807-3107bor-2020.vol34.0008

Submitted: August 5, 2019

Accepted for publication: December 10, 2019

Last revision: January 13, 2020

\begin{abstract}
This study aimed to investigate the effects of chronic restraint stress (RS) and a high-fat diet (HFD) on the osseointegration of titanium implants in a rat model. After the surgical insertion of titanium implants into the metaphysis of the tibial bone, the rats were randomly divided into four equal groups ( $\mathrm{n}=8$ each): control (CNT), restraint stress (RS), high-fat diet (HFD), and restraint stress plus high fat diet (RS-HFD). CNT: Rats received no further treatment during the 92-day experimental period. RS: Stress was applied to the rats beginning from two days after the implant surgery for one hour per day for the first 30 days, two hours per day for the next 30 days, and three hours per day for the last 30 days. HFD: Rats were fed a HFD for the following 90 days starting two days after surgery. RS-HFD: Rats were fed a HFD and RS was applied to rats for the following 90 days, starting two days after surgery. At the end of the experimental period, the rats were euthanized, and the implants and surrounding bone tissues were removed for histological analysis. Statistical analysis was performed by one way ANOVA and Bonferrroni tests. There were no significant differences in the bone-implant connection levels between the groups ( $p>0.05)$, but in the HFD and RS-HFD groups, the bone filling ratios were found to be lower compared with the controls $(p<0.05)$ The data analyzed in this study suggest that an HFD with or without chronic RS adversely affected bone tissue in the rats during the 90-day osseointegration period.
\end{abstract}

Keywords: Hydrocortisone; Diet, High-Fat; Osseointegration; Bone-Implant Interface.

\section{Introduction}

Atherosclerosis, vascular calcification and osteoporosis are major health problems of the aging population. High-fat diet (HFD)-induced hyperlipidemia has adverse effects on the cardiovascular system, including vascular problems. Under hyperlipidemic conditions, protein-bound lipid particles pass through the endothelial wall into the subendothelial field, where they are caught and oxidatively modified by reactive oxygen species produced by metabolically-active neighboring smooth muscle cells and macrophages. A similar cycle takes place in human osteoporotic bone, with oxidized protein-bound lipid particles collecting in the perivascular and subendothelial fields. Osteoblasts also have the ability to oxidatively 
modify protein-bound lipid particles, and oxidized lipid products have been detected in the bone marrow of hyperlipidemic mice. ${ }^{1,2,3,4}$

According to the National Health and Nutrition Examination Survey, $63 \%$ of osteoporotic patients have hyperlipidemia. Moreover, numerous studies have reported that obesity is a risk factor for osteoporosis in humans. Epidemiological studies reported a statistically significant inverse relationship between serum cholesterol levels and bone mineral parameters and density, independent of age and body mass index. HFD consumption is also associated with a decrease in bone mineral content and density in experimental animal studies. Additionally, whether due to a genetic or dietary abnormality, excessive lipid-derived reactive oxygen species reduce osteoblastic differentiation in vitro. In addition, oxidized lipids induce osteoclastogenesis and decrease parathyroid hormone and bone morphogenetic protein-2 signaling. Experimental studies have reported that hyperlipidemia induces bone loss in mice..$^{1,2,3}$

Stress significantly affects a large proportion of the population worldwide, regardless of age, sex, or social class. The scientific community believes stress to be one of the most important risk factors for the onset of numerous diseases. Indeed, chronic stress can negatively affect both physical and mental health. Stress activates the hypothalamic-pituitary-adrenal pathway and thereby increases the amount of corticotrophin-releasing hormone secreted by the hypothalamic paraventricular nucleus. This mechanism, in turn, stimulates the release of adrenal corticosteroids by secreting adrenocorticotropin from the anterior pituitary gland. ${ }^{5,6,7}$

Wound healing is known to be affected by various conditions, including chronic and acute stress. Although the mechanisms that cause stress to disrupt the process of wound healing have not yet been fully elucidated, the disruption may occur due to stress activating the hypothalamic-pituitary-adrenal axis. In addition, some experimental animal studies have reported that glucocorticoids delay soft-tissue wound healing and thereby adversely affect bone-implant connections. ${ }^{78,9,0}$ Dental implant-supported prostheses are a commonly used and scientifically accepted treatment option for both partial and complete edentulism. However, the systemic condition of the patient, the quality of the bone, the shape and surface characteristics of the implant, and smoking habits are all important factors in relation to the success of dental implant therapy. Although there is a growing body of evidence suggesting that chronic stress negatively affects wound healing, the effects of stress on the bone-implant connection (BIC) have not yet been fully investigated in the literature and hence remain controversial. ${ }^{8,9,11,12}$

The aim of this study was to investigate the effects of an HFD and chronic stress on the osseointegration of titanium implants in the tibial bones of rats.

\section{Methodology}

\section{Animals and experimental design}

The number of animals required for the experiments was determined by power analysis; $8 \%$ deviation, type 1 error $(\alpha) 0.05$ and type 2 error $(\beta)($ Power $=0.80)$, and for experiments in which the animals were divided into groups, at least 7 animals were required in each group.

All the experimental and surgical procedures involved in this study were conducted at the Firat University Experimental Research Center in Elazig, Turkey. Approval for the study was granted by the Firat University Animal Experimental Ethics Council (Protocol Number: 2016/77, Date: July 13, 2016). Further, the recommendations of the Declaration of Helsinki regarding the protection of laboratory research animals were stringently followed. In this study, 32 healthy adult female Sprague Dawley rats aged 2.5-3 months were used. On the first day of the experimental period, the average body weight of the rats was 220-230 grams. The rats were kept in plastic cages, and their temperatures were checked daily. Throughout the experimental period, the rats had free access to food and water, and they experienced a cycle of 12 hours of dark and 12 hours of light.

Rats with the same estrus period were included in the study. Titanium implants (Bioinfinity Dental Implant System, Avrupa Dental Implant Corporation, Istanbul, Turkey) were surgically inserted into the tibial bones of the rats. Two days after the surgical 
procedures, the rats were randomly divided into four groups, $\mathrm{n}=8$ per group, with similar mean weights in each group: control (CNT) group, restraint stress (RS) group, high-fat diet (HFD) group, and restraint stress and high-fat diet (RS-HFD) group. In the CNT group, the rats received no further treatment over the 92-day experimental period following implant surgery. In the RS group, restraint stress was applied for one hour per day throughout the first 30 days after the second postoperative day, two hours per day throughout the next 30 days, and three hours per day throughout the last 30 days of the 92-day experimental period. ${ }^{8}$ In the HFD group, the rats were fed an HFD for 90 days after the second postoperative day. ${ }^{13}$ In the RS-HFD group, restraint stress was applied for one hour per day throughout the first 30 days after the second postoperative day, two hours per day throughout the next 30 days, and three hours per day throughout the last 30 days of the experimental period. ${ }^{8}$

\section{Surgical procedures}

Ketamine hydrochloride $(50 \mathrm{mg} / \mathrm{kg}$, Ketasol, Richter Pharma AG, Wels, Austria) and xylazine (5 mg/kg, Rompun, Bayer, Germany) were intramuscularly injected into the rats, and general anesthesia was achieved. All the surgical procedures were performed under sterile conditions. After the rats were anesthetized, the surgical site was washed with povidone-iodine and shaved. A 15-mm-long incision was made to the right tibial crest, the soft tissues were dissected and incised, and the tibial metaphyseal bone was exposed. Implant sockets were created using appropriate drills with saline perfusion. The machine-surfaced titanium implants were then installed into the metaphyseal part of the tibial bone, and primary stabilization was achieved. ${ }^{14}$ The titanium implants were $4 \mathrm{~mm}$ in length and $2.5 \mathrm{~mm}$ in diameter. ${ }^{14}$ Following the placement of the titanium implants, the flap was returned to the original position, and the fascia, subcutaneous tissue, and skin were sutured using 4-0 polyglactin sutures. In order to prevent pain and infection, an antibiotic $(50 \mathrm{mg} / \mathrm{kg}$ penicillin) and an analgesic $(0.1 \mathrm{mg} / \mathrm{kg}$ tramadol hydrochloride) were intramuscularly injected into each rat daily for three days following the surgical procedures. All the surgical procedures were performed atraumatically by the same researcher.

\section{Restraint stress application}

Restraint stress was applied to the rats in the RS group using a special device. The rats were placed in polyvinylchloride tubing according to their sizes, and holes were drilled in the tubes at the level of the rats' noses to allow them to breathe. This restraint procedure was used throughout the experimental period. ${ }^{8,14}$

\section{Animal feeding}

The control group was fed with a standard chow diet. The HFD group was fed with a chow diet supplemented with $42 \%$ of the calories as fat. ${ }^{13}$

\section{Histological and biochemical analysis}

No fatal or nonfatal complications (such as wound formation or wound infection) were encountered during the experimental period generally. Only one rat from the RS group and one rat from the RS-HFD group died. At the end of the experiment, the rats were euthanized under deep anesthesia, and their titanium implants along with the surrounding bone tissues were harvested and fixed in 10\% formalin solution for one week. Non-decalcified histological analysis was performed at the Faculty of Dentistry, Research Laboratory, Erciyes University, Kayseri, Turkey. For histological analysis, the titanium implants and surrounding bone tissues were embedded in 2-hydroxyethylmethacrylate and sectioned using an Exakt ${ }^{\circledR}$ microtome (EXACT Technologies Inc., Oklahoma City, USA). After the specimens were cut across the middle, each section was ground using the Exakt ${ }^{\circledR}$ grinder to obtain $50-\mu$ m-thick sections for performing light microscopy analysis. Histological staining for BIC and bone filling (BF) analysis was performed using toluidine blue. BIC and BF were analyzed using a light microscope and an image analyzer at the Department of Microbiology Laboratory, Faculty of Medicine, Firat University, Elazig, Turkey. Blinded and calibrated histomorphometry was performed using a stereological software system (Nikon, Tokyo, Japan). The BIC ratio (\%) of each specimen was calculated as the ratio of implant surface 
directly touching the bone to the total implant surface length. ${ }^{1,11,12}$ Bone filling ratio (\%) was calculated by measuring the bone-filled areas at a distance of $0.5 \mathrm{~mm}$ from the titanium implant (mesial, distal and apical portions of the implants). ${ }^{15}$ Under deep anesthesia, blood samples were collected by means of cardiac puncture without anticoagulant in order to measure the cortisol, serum triglyceride, glucose, very low density lipoprotein (VLDL), aspartate aminotransferase (AST), and alanine aminotransferase (ALT) levels. ${ }^{16}$ Biochemical data were measured for each rat in the biochemistry laboratory of Firat University Faculty of Medicine. To guard against fluctuations in serum cortisol levels due to the circadian rhythm, all blood samples were collected at 10:00 AM. ${ }^{16}$

\section{Statistical analysis}

Statistical analysis was performed using SPSS 23.0 for Windows software (IBM SPSS Statistics for Windows, Armonk, NY, USA). Data for each group are expressed as mean \pm standard deviation. Differences between the groups were detected using one-way ANOVA. Bonferroni honestly significant difference test was used to determine the group responsible for these differences, and $P<0.05$ was considered statistically significant.

\section{Results}

During the experimental period, one rat from the RS group and one rat from the RS-HFD group died. Hence, the study was completed with seven rats in these groups. Due to improper preparation (for reasons such as separation of the implant from tissue and separation of bone from the histologic preparation), one specimen was eliminated from the HFD group and one from the control group. In the biochemical analysis, cortisol levels below $0.5 \mu \mathrm{g} / \mathrm{dL}$ were accepted as $0 \mu \mathrm{g} / \mathrm{dL} \cdot{ }^{14}$ Cortisol levels were significantly higher in the rats in the RS and RS-HFD groups than in the rats in the control and HFD groups $(\mathrm{p}<0.05)$. However, cortisol levels were not significantly different between the rats in the control and the HFD groups (Table 1$)(p>0.05){ }^{14}$

Higher levels of triglyceride and VLDL were detected in both HFD and RS-HFD groups compared with the RS and control groups $(\mathrm{p}<0.05) \cdot{ }^{17}$ No significant differences were detected between the groups in terms of glucose, AST, ALT levels or weight (Table 1) ( $p>0.05$ ).

Although the bone-implant connection ratio was higher in the control group numerically (Figure 1) compared to the experimental groups (Figures 2, 3 and 4), no statistically significant differences were observed in the BIC ratios of the rats between any of the groups (Table 2) $(p>0.05)$.

$\mathrm{BF}$ ratios were found to be significantly lower in the HFD (Figure 3) and RS-HFD (Figure 4) groups compared with the controls (Figure 1) (Table 3$)(p<0.05)$.

\section{Discussion}

Adipose tissue is not an inert organ with the sole function of storing energy; it also has metabolic functions, among which are secretion of proteins that are involved in bone metabolism. Indeed, an HFD has a greater impact on bone metabolism due to the resultant endocrine stimulus rather than the mechanical stimulus, regardless of a subject's weight. ${ }^{18,19,20}$ Several mechanisms have been proposed to explain HFD-induced osteoclastogenesis, including elevated levels of the pro-inflammatory cytokines IL-1 and TNF derived from adipose tissue macrophages in the blood, increased RANKL expression in the bones, and decreased expression of the anti-osteoclastogenic cytokine IL-10. ${ }^{21,22,23,24,25,26}$ In a previous study, the authors reported that HFD-induced obesity during growth not only triggers mandibular osteoporosis but also increases the risk of spontaneous periodontal disease. ${ }^{25}$ In a study conducted by Lac et al. ${ }^{26}$, mice fed an HFD during the early developmental period had lower bone mineral content and bone mineral density. In addition, it has been reported that there is a negative correlation between the visceral fat ratio and bone mineral density. Moreover, Lu et al. ${ }^{26}$ reported similar results in an in vivo study in young male rats. They reported that an HFD significantly decreased the bone mineral content and the trabecular bone area compared with those of rats that were fed a normal diet. Pirih et al. ${ }^{2}$ reported that oxidative lipids and/or hyperlipidemia negatively affected the mechanical strength and disrupted the regeneration process of the femoral bone of rats in the study they performed. 
Table 1. Biochemical parameters of the groups.

\begin{tabular}{|c|c|c|c|}
\hline Parameters & Groups & Mean \pm Std. Deviation & p-value* \\
\hline \multirow{5}{*}{ VLDL (mg/dL) } & Control $(n=7)$ & $7,37 \pm 2,02$ & \multirow{5}{*}{$<0,05$} \\
\hline & Stress $(\mathrm{n}=7)$ & $7,17 \pm 1,92$ & \\
\hline & Stress $+\mathrm{HFD}$ & $1774+57$ & \\
\hline & $(n=7)^{a, b, \alpha}$ & $1 /, 14 \pm 3,11$ & \\
\hline & $\operatorname{HFD}(n=7)^{a, b, a}$ & $17,20 \pm 7,45$ & \\
\hline \multirow{5}{*}{ Triglycerides (mg/dL) } & Control $(n=7)$ & $36,86 \pm 10,11$ & \multirow{5}{*}{$<0,05$} \\
\hline & Stress $(n=7)$ & $35,86 \pm 9,62$ & \\
\hline & Stress + HFD & $0571+2005$ & \\
\hline & $(n=7)^{a, b, \alpha}$ & $85,11 \pm 28,85$ & \\
\hline & $\operatorname{HFD}(n=7)^{a, b, \alpha}$ & $86,00 \pm 37,27$ & \\
\hline \multirow{4}{*}{ Glucose (mg/dL) } & Control $(n=7)$ & $128 \pm 9,17$ & \multirow{4}{*}{$>0,05$} \\
\hline & Stress $(n=7)$ & $123,14 \pm 18,1$ & \\
\hline & Stress $+\operatorname{HFD}(n=7)$ & $122,57 \pm 20,7$ & \\
\hline & $\operatorname{HFD}(n=7)$ & $120,86 \pm 12,52$ & \\
\hline \multirow{5}{*}{ Cortisol ( $\mu / d L)$} & Control $(n=7)$ & $0,00 \pm 00$ & \multirow{5}{*}{$<0,05$} \\
\hline & Stress $(n=7)^{a, c, \alpha}$ & $0,39 \pm 0,27$ & \\
\hline & Stress + HFD & $016+027$ & \\
\hline & $(n=7)^{a, c, \alpha}$ & $0,40 \pm 0,21$ & \\
\hline & $\operatorname{HFD}(n=7)$ & $0,00 \pm 00$ & \\
\hline \multirow{4}{*}{ AST (U/L) } & Control $(n=7)$ & $254,43 \pm 51,6$ & \multirow{4}{*}{$>0,05$} \\
\hline & Stress $(n=7)$ & $270,29 \pm 49,92$ & \\
\hline & Stress + HFD $(n=7)$ & $275,29 \pm 31,64$ & \\
\hline & $\operatorname{HFD}(n=7)$ & $240 \pm 68,78$ & \\
\hline \multirow{4}{*}{ ALT (U/L) } & Control $(n=7)$ & $72,86 \pm 20,22$ & \multirow{4}{*}{$>0,05$} \\
\hline & Stress $(n=7)$ & $76,57 \pm 21$ & \\
\hline & Stress $+\operatorname{HFD}(n=7)$ & $76,86 \pm 9,44$ & \\
\hline & $\operatorname{HFD}(\mathrm{n}=7)$ & $78,29 \pm 18,55$ & \\
\hline \multirow{4}{*}{ Weight (g) (3 Months) } & Control $(n=7)$ & $284,29 \pm 27,68$ & \multirow{4}{*}{$>0,05$} \\
\hline & Stress $(\mathrm{n}=7)$ & $280 \pm 27,92$ & \\
\hline & Stress + HFD $(n=7)$ & $278,86 \pm 27,75$ & \\
\hline & $\operatorname{HFD}(\mathrm{n}=7)$ & $300,64 \pm 27,5$ & \\
\hline \multirow{4}{*}{ Weight (g) (Initial) } & Control $(n=7)$ & $225 \pm 2,78$ & \multirow{4}{*}{$>0,05$} \\
\hline & Stress $(n=7)$ & $227 \pm 2,77$ & \\
\hline & Stress + HFD $(n=7)$ & $224,71 \pm 2,14$ & \\
\hline & $\mathrm{HFD}(\mathrm{n}=7)$ & $224,43 \pm 27,5$ & \\
\hline
\end{tabular}

"One Way ANOVA; ${ }^{\alpha}$ The Bonferroni test was used in comparisons between groups; ${ }^{a}$ Statistically significant difference compared with the controls; bStatistically significant difference compared with the stress group; CStatistically significant difference compared with the HFD group; VLDL: very low density lipoprotein; HFD: high fat diet; AST: aspartate aminotransferase; ALT: alanine aminotransferase.

Additionally, they reported that in the HFD group, the cortical bone volume fraction in the femoral bone showed significantly greater decreases compared with rats that were fed a normal diet. Keuroghlian et al. ${ }^{28}$ reported that in atherosclerosis-susceptible C57BL/6J male mice fed an HFD, both the amount and strength of bone-implant contact in the femurs were reduced and implant loss significantly increased. These results support the hypothesis that an HFD may reduce osseointegration and lead to adverse outcomes in dental implant therapy. In this study, the BIC ratios of the groups did not show a statistically significant difference. However, in both HFD groups (HFD and RS-HFD) the BF ratios were found to be 


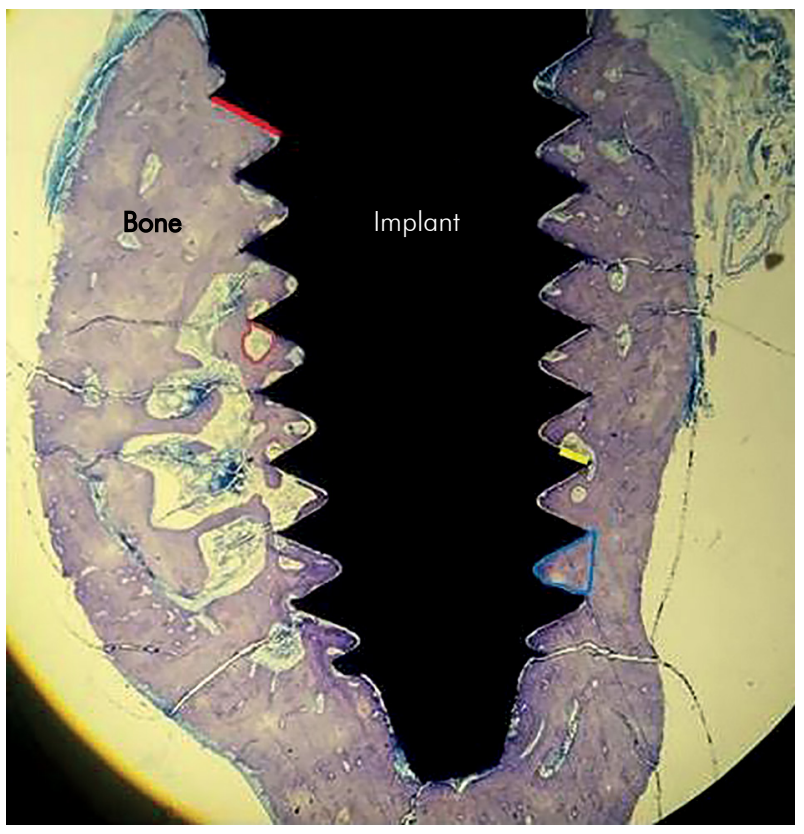

Figure 1. Non-decalcified histologic images of the Control Group, (40 times magnification, tolvidine blue staining). Yellow Line: Implant surface not contacting bone $(\alpha)$, Red Line: Implant surface in contact with bone $(\beta)$, Total implant surface: $£$, Bone Implant Contact Ratio $(\%): £-\alpha(\beta) / £$. Bone filling detected by measuring the bone-filled areas at a distance of $0.5 \mathrm{~mm}$ from the implant (mesial, distal and apical portion of implants). Bone-filled areas (à: Blue Line), non-bone areas (ĕ: Brown

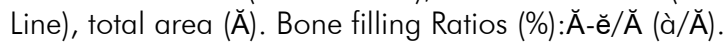

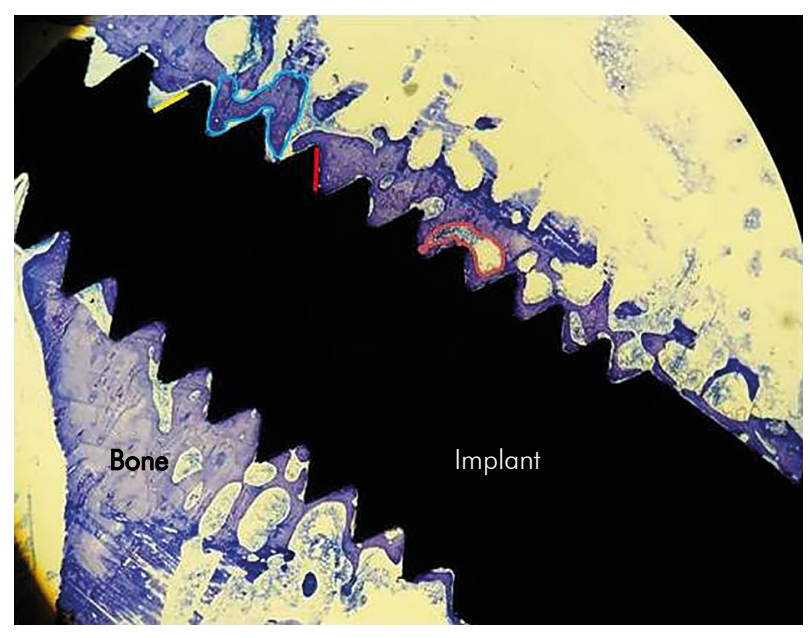

Figure 2. Non-decalcified histologic images of the Restraint Stress Group, (40 times magnification, tolvidine blue staining). Yellow Line: Implant surface not contacting bone $(\alpha)$, Red Line: Implant surface in contact with bone ( $\beta)$, Total implant surface: $£$, Bone Implant Contact Ratio (\%): $£-\alpha(\beta) / £$. Bone filling detected by measuring the bone-filled areas at a distance of $0.5 \mathrm{~mm}$ from the implant (mesial, distal and apical portion of implants). Bone-filled areas (à: Blue Line), non-bone areas (ě: Brown Line),

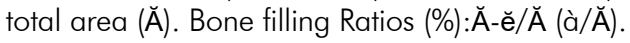

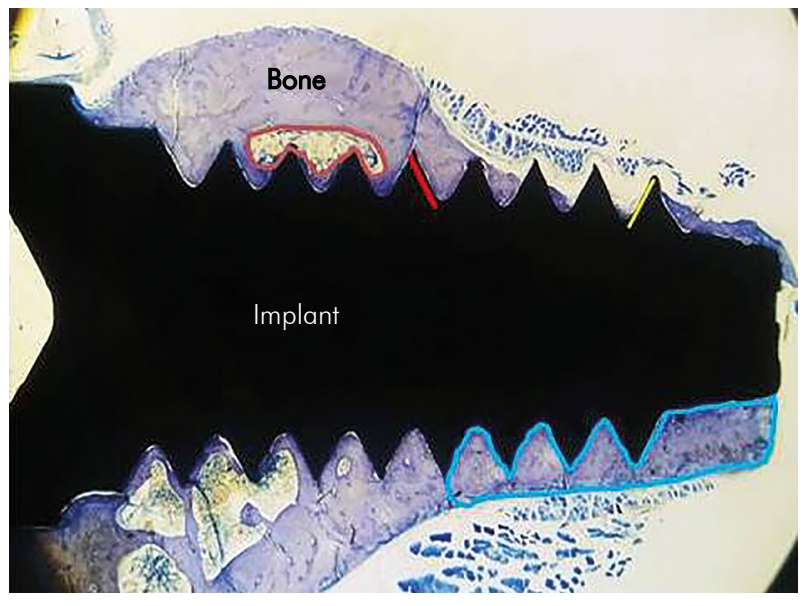

Figure 3. Non-decalcified histologic images of the High Fat Diet Group, (40 times magnification, toluidine blue staining). Yellow Line: Implant surface not contacting bone $(\alpha)$, Red Line: Implant surface in contact with bone ( $\beta)$, Total implant surface: $£$, Bone Implant Contact Ratio $(\%): £-\alpha(\beta) / £$. Bone filling detected by measuring the bone-filled areas at a distance of $0.5 \mathrm{~mm}$ from the implant (mesial, distal and apical portion of implants). Bone-filled areas (à: Blue Line), non-bone areas (ě: Brown Line), total area $(\breve{A})$. Bone filling Ratios (\%): $\breve{A}-\mathrm{e} / \breve{A}(a ̀ / \breve{A})$.

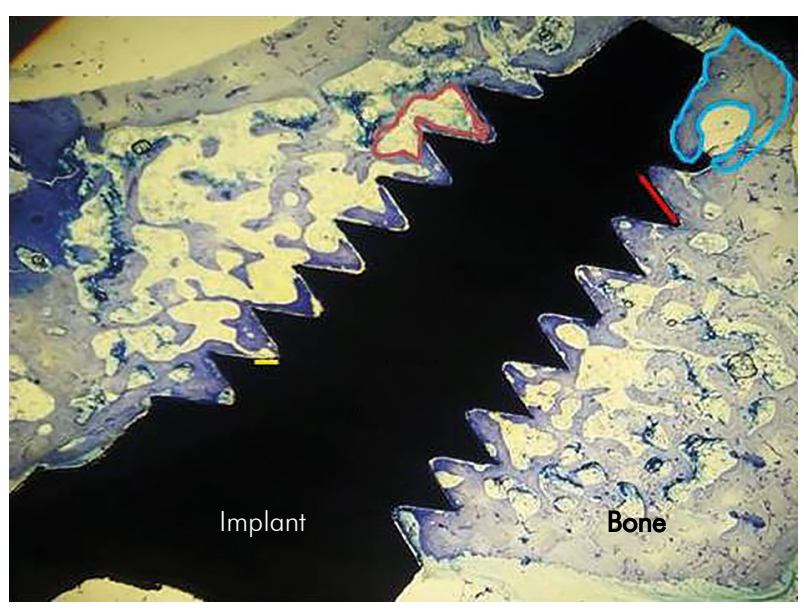

Figure 4. Non-decalcified histologic images of the High Fat Diet-Restraint Stress Group, (40 times magnification, toluidine blue staining). Yellow Line: Implant surface not contacting bone $(\alpha)$, Red Line: Implant surface in contact with bone $(\beta)$, Total implant surface: $£$, Bone Implant Contact Ratio $(\%): £-\alpha(\beta) / £$. Bone filling detected by measuring the bone-filled areas at a distance of $0.5 \mathrm{~mm}$ from the implant (mesial, distal and apical portion of implants). Bone-filled areas (à: Blve Line), non-bone areas (ĕ: Brown Line), total area $(\breve{A})$. Bone filling Ratios (\%): $\breve{A}-\check{e} / \check{A}$ (à/Ă).

lower compared with the controls. The BF results of this study support the results of the study of Keuroglian et al., ${ }^{28}$ who reported that more than one 
Table 2. Bone-implant connection (BIC) ratios (\%) of the groups.

\begin{tabular}{lcc}
\hline Groups & BIC (\%) Mean \pm Std deviation & p-value* \\
\hline Control $(n=7)$ & $71,80 \pm 9,17$ & \\
Stress $(n=7)$ & $62,71 \pm 5,41$ & \\
Stress + HFD $(n=7)$ & $64,88 \pm 6,13$ & $>0,05$ \\
HFD $(n=7)$ & $69,16 \pm 6,40$ & \\
\hline
\end{tabular}

HFD: high fat diet, ${ }^{*}$ one way ANOVA.

Table 3. Bone filling ratios (\%) of the groups.

\begin{tabular}{lcc}
\hline Groups & Bone Filling (\%) & p-value* \\
\cline { 2 - 2 } & Mean \pm Std. Deviation & -valu \\
\hline Control $(n=7)$ & $55,29 \pm 11,09$ & \\
Stress $(n=7)$ & $45,14 \pm 5,24$ & \\
Stress + HFD $(n=7)$ & $42,86 \pm 6,07^{\alpha, 1} \quad p<0,05(0,016)$ \\
HFD $(n=7)$ & $43,86 \pm 5,61^{\alpha, 2}$ & \\
\hline
\end{tabular}

HFD: high fat diet, *one way ANOVA. ${ }^{*}$ Statistically significant difference compared with controls ${ }^{\beta}$; $(p<0.05 \beta$ : Bonferroni test, 1: p: 0.026, 2: p: 0.048).

mechanism is effective in reducing BIC in HFD-fed rats. Cell-level hyperlipidemic conditions lead to the inhibition of osteogenic signaling, the reduction of mature osteoblast formation, increased production of molecular markers in bone remodeling, enhanced osteoclast differentiation and activity, and increased bone resorption. Additionally in this study VLDL triglyceride levels in the subjects fed an HFD were found to be significantly higher than those in the control group..$^{17}$ However, glucose, liver parameters and weight measurement did not differ between the groups. These results may be indicative of the effect of an HFD on blood fat metabolism.

The pathophysiological relationship between stress and wound healing may be related to an increase in glucocorticoid levels. Cortisol is the most abundant glucocorticoid species in rats and is thought to be a useful marker of rodents' stress levels. In this study, the higher levels of plasma cortisol and lower weights observed in the stressed rats compared to those of the rats in the control group is an indication of the importance of stress management. These results also show that, during the osseointegration period, the rats in the chronic stress group did not adapt to the stress protocol. ${ }^{9,29}$ The cortisol levels can be evaluated as physiological data related to the presence of stress, and this study shows that the experimental stress model increases cortisol in the bloodstream.,29 Stress can suppress or stimulate the immune system, and activates the hypothalamic-pituitaryadrenal pathway. Activation of this pathway results in the release of neuroendocrine and adrenal corticosteroid hormones, making people susceptible to disease. The immuno-inflammatory response is a key factor in the mechanism of periodontitis and periimplantitis. ${ }^{30,31}$ Research into the relationship between bone tissue and stress has shown that long-term administration of glucocorticoids increases osteoclast activity, suppresses osteoblastic activity, decreases bone mineral density, and is associated with the development of osteonecrosis. ${ }^{32,33,34,35,36}$ Conte Neto et al. ${ }^{9}$ reported that the stressed rats in their experimental study had more areas of empty osteocytic lacunae than the controls. The association between glucocorticoids and osteonecrosis induction pathways may explain these findings. ${ }^{34,35,36}$ Siqueira et al. ${ }^{8}$ reported that chronic stress may impair osseointegration by affecting the initial phase of bone healing in the rat mandible. According to Siqueira et al. ${ }^{8}$, in the early stages of bone healing, they detected less osseointegration of titanium implants in a stressed group than in a control group. In their experimental animal study, Siqueira et al. ${ }^{8}$ found that stress inhibited bone matrix formation and the activity of bone-forming cells, as well as the formation of collagenous fibers. They reported that 18 days of high-intensity chronic stress destroyed the healing of immature tissues. At the end of the 18-day experimental period, the osseointegration level was found to be significantly lower in the stressed group than in the control group. ${ }^{8}$ In a 28-day experimental osseointegration study, Conte Neto et al. ${ }^{9}$ detected an impairment of bone metabolism, represented by the low levels of bone-specific alkaline phosphatase and bone area fraction occupancy values in a chronic stress group. Additionally Bozoglan et al. reported that the application of 4 hours of restraint stress daily over a 28 day osseointegration period impaired the BIC in rat tibias. We examined the BIC 92 days after the surgical procedure and could not detect any statistically significant difference in BIC between the groups. However BF values in the RS-HFD group were found to be lower compared with the controls. 
Another result observed in our study was that stress affected BF if it was applied to rats fed the HFD diet, while in contrast RS alone did not affect the BIC in this study. These results are valuable in revealing the negative relationship between stress, an HFD and bone tissue metabolism.

The present study has several limitations. First, molecular mechanisms underlying the association between RS and bone tissue metabolism could not be fully explained because of the method used in this study. Second, although in vivo studies are vital for understanding pathways underlying RS-induced pathologies, results of these studies can only be used to estimate corresponding pathways in humans. Third, in this study, we were unable to evaluate the survival rate of titanium implants or the success of long-term bone-implant connections. Fourth, long bones such as the tibia and femur have different osteogenic properties to the jaw bones (mandible-maxilla) and therefore may respond differently to RS application.

\section{Conclusion}

Within the limitations of this study, RS and an HFD did not affect the BIC. However an HFD alone or an HFD with RS adversely affected the bone filling in peri-implant bone tissues. Further clinical and experimental studies are needed to clarify the relationships between osseointegration and bone metabolism, a high fat diet, and stress.

\section{Acknowledgement}

This study was supported by Firat University Scientific Research Project Foundation Department, Elazig, Turkey with the project number DHF.16.05. The authors wish to thanks Bioinfinity Dental ImplantSystem, Avrupa Dental Implant Corporation, Istanbul, Turkey for manufacturing and providing the titanium implants.

Note: Some of the information presented in this study was presented as an oral presentation at the $25^{\text {th }}$ International Scientific Congress of Turkish Dental Association at 5-7 September 2019, Istanbul, Turkey.

\section{References}

1. Dündar S, Yaman F, Ozupek MF, Saybak A, Gul M, Asutay F, et al. The effects of high-fat diet on implant osseointegration: an experimental study. J Korean Assoc Oral Maxillofac Surg. 2016 Aug;42(4):187-92. https://doi.org/10.5125/jkaoms.2016.42.4.187

2. Pirih F, Lu J, Ye F, Bezouglaia O, Atti E, Ascenzi MG, et al. Adverse effects of hyperlipidemia on bone regeneration and strength. J Bone Miner Res. 2012 Feb;27(2):309-18. https://doi.org/10.1002/jbmr.541

3. Tintut Y, Morony S, Demer LL. Hyperlipidemia promotes osteoclastic potential of bone marrow cells ex vivo. Arterioscler Thromb Vasc Biol. 2004 Feb;24(2):e6-10. https://doi.org/10.1161/01.ATV.0000112023.62695.7f

4. Brodeur MR, Brissette L, Falstrault L, Ouellet P, Moreau R. Influence of oxidized low-density lipoproteins (LDL) on the viability of osteoblastic cells. Free Radic Biol Med. 2008 Feb;44(4):506-17. https://doi.org/10.1016/i.freeradbiomed.2007.08.030

5. Semenoff-Segundo A, Porto AN, Semenoff TA, Cortelli JR, Costa FO, Cortelli SC, et al. Effects of two chronic stress models on ligature-induced periodontitis in Wistar rats. Arch Oral Biol. 2012 Jan;57(1):66-72. https://doi.org/10.1016/j.archoralbio.2011.07.014

6. Takada T, Yoshinari N, Sugiishi S, Kawase H, Yamane T, Noguchi T. Effect of restraint stress on the progression of experimental periodontitis in rats. J Periodontol. 2004 Feb;75(2):306-15. https://doi.org/10.1902/jop.2004.75.2.306

7. Sapolsky RM. Stress, glucocorticoids, and damage to the nervous system: the current state of confusion. Stress. 1996 Jul;1(1):1-19. https://doi.org/10.3109/10253899609001092

8. Siqueira CR, Semenoff TA, Palma VC, Borges ÁH, Silva NF, Segundo AS. Effect of chronic stress on implant osseointegration into rat's mandible. Acta Cir Bras. 2015 Sep;30(9):598-603. https://doi.org/10.1590/S0102-865020150090000003

9. Conte Neto N, Andrade CR, Spolidorio LC, Planeta CS, Cruz FC, Bastos AS, et al. Effects of chronic stress and alendronate therapy on the osseointegration of titanium implants. Clin Implant Dent Relat Res. 2014 Oct;16(5):762-71. https://doi.org/10.1111/cid.12046

10. Marucha PT, Kiecolt-Glaser JK, Favagehi M. Mucosal wound healing is impaired by examination stress. Psychosom Med. 1998 May-Jun;60(3):362-5. https://doi.org/10.1097/00006842-199805000-00025

11. Dundar S, Yaman F, Gecor O, Cakmak O, Kirtay M, Yildirim TT, et al. Effects of Local and Systemic Zoledronic Acid Application on Titanium Implant Osseointegration: An Experimental Study Conducted on Two Surface Types. J Craniofac Surg. 2017 Jun;28(4):935-8. https://doi.org/10.1097/SCS.0000000000003568 
Dundar S, Bozoglan A, Bulmus O, Tekin S, Yildirim TT, Kirtay M, et al.

12. Dundar S, Yaman F, Bozoglan A, Yildirim TT, Kirtay M, Ozupek MF, et al. Comparison of Osseointegration of Five Different Surfaced Titanium Implants. J Craniofac Surg. 2018 Oct;29(7):1991-5. https://doi.org/10.1097/SCS.0000000000004572

13. Sahin K, Tuzcu M, Orhan C, Agca CA, Sahin N, Guvenc M, et al. The effects of chromium complex and level on glucose metabolism and memory acquisition in rats fed high-fat diet. Biol Trace Elem Res. 2011 Nov;143(2):1018-30. https://doi.org/10.1007/s12011-010-8905-9

14. Bozoglan A, Dundar S, Yildirim TT, Bulmus O, Ertugrul AS, Bozoglan MY, et al. Effects of different levels of restraint stress on bone-implant contact. J Craniofac Surg. 2019 Jun;30(4):1294-7. https://doi.org/10.1097/SCS.0000000000005104

15. Nociti Júnior FH, Cesar Neto JB, Carvalho MD, Sallum EA, Sallum AW. Intermittent cigarette smoke inhalation may affect bone volume around titanium implants in rats. J Periodontol. 2002 Sep;73(9):982-7. https://doi.org/10.1902/jop.2002.73.9.982

16. Padgett DA, Marucha PT, Sheridan JF. Restraint stress slows cutaneous wound healing in mice. Brain Behav Immun. 1998 Mar;12(1):64-73. https://doi.org/10.1006/brbi.1997.0512

17. Nunes RCA, Chiba FY, Pereira AG, Pereira RF, Mattera MSLC, Ervolino E, et al. Effect of sodium fluoride on bone biomechanical and histomorphometric parameters and on insulin signaling and insulin sensitivity in ovariectomized rats. Biol Trace Elem Res. 2016 Sep;173(1):144-53. https://doi.org/10.1007/s12011-016-0642-2

18. Yamanaka JS, Yanagihara GR, Carlos BL, Ramos J, Brancaleon BB, Macedo AP, et al. A high-fat diet can affect bone healing in growing rats. J Bone Miner Metab. 2018 May;36(3):255-63. https://doi.org/10.1007/s00774-017-0837-4

19. Vendrell J, Broch M, Vilarrasa N, Molina A, Gómez JM, Gutiérrez C, et al. Resistin, adiponectin, ghrelin, leptin, and proinflammatory cytokines: relationships in obesity. Obes Res. 2004 Jun;12(6):962-71. https://doi.org/10.1038/oby.2004.118

20. Greco EA, Lenzi A, Migliaccio S. The obesity of bone. Ther Adv Endocrinol Metab. 2015 Dec;6(6):273-86. https://doi.org/10.1177/2042018815611004

21. Shu L, Beier E, Sheu T, Zhang H, Zuscik MJ, Puzas EJ, et al. High-fat diet causes bone loss in young mice by promoting osteoclastogenesis through alteration of the bone marrow environment. Calcif Tissue Int. 2015 Apr;96(4):313-23. https://doi.org/10.1007/s00223-015-9954-z

22. Kyung TW, Lee JE, Phan TV, Yu R, Choi HS. Osteoclastogenesis by bone marrow-derived macrophages is enhanced in obese mice. J Nutr. 2009 Mar;139(3):502-6. https://doi.org/10.3945/jn.108.100032

23. Woo DG, Lee BY, Lim D, Kim HS. Relationship between nutrition factors and osteopenia: effects of experimental diets on immature bone quality. J Biomech. 2009 May;42(8):1102-7. https://doi.org/10.1016/i.jbiomech.2009.02.020

24. Graham LS, Tintut Y, Parhami F, Kitchen CM, Ivanov Y, Tetradis S, et al. Bone density and hyperlipidemia: the T-lymphocyte connection. J Bone Miner Res. 2010 Nov;25(11):2460-9. https://doi.org/10.1002/ibmr.148

25. Fujita Y, Maki K. High-fat diet-induced obesity triggers alveolar bone loss and spontaneous periodontal disease in growing mice. BMC Obes. 2016 Jan;3:1. https://doi.org/10.1186/s40608-016-0082-8

26. LuXM, Zhao H, Wang EH. A high-fat diet induces obesity and impairs bone acquisition in young male mice. Mol Med Rep. 2013 Apr;7(4):1203-8. https://doi.org/10.3892/mmr.2013.1297

27. Lac G, Cavalie H, Ebal E, Michaux O. Effects of a high fat diet on bone of growing rats. Correlations between visceral fat, adiponectin and bone mass density. Lipids Health Dis. 2008 Apr;7(1):16. https://doi.org/10.1186/1476-511X-7-16

28. Keuroghlian A, Barroso AD, Kirikian G, Bezouglaia O, Tintut Y, Tetradis S, et al. The effects of hyperlipidemia on implant osseointegration in the mouse femur. J Oral Implantol. 2015 Apr;41(2):e7-11. https://doi.org/10.1563/AAID-JOI-D-13-00105

29. Park MH, Rehman SU, Kim IS, Choi MS, Yoo HH. Stress-induced changes of neurosteroid profiles in rat brain and plasma under immobilized condition. J Pharm Biomed Anal. 2017 May;138(138):92-9. https://doi.org/10.1016/i.jpba.2017.02.007

30. Botelho J, Machado V, Mascarenhas P, Rua J, Alves R, Cavacas MA, et al. Stress, salivary cortisol and periodontitis: A systematic review and meta-analysis of observational studies. Arch Oral Biol. 2018 Dec;96:58-65. https://doi.org/10.1016/i.archoralbio.2018.08.016

31. Cakmak O, Tasdemir Z, Aral CA, Dundar S, Koca HB. Gingival crevicular fluid and saliva stress hormone levels in patients with chronic and aggressive periodontitis. J Clin Periodontol. 2016 Dec;43(12):1024-31. https://doi.org/10.1111/icpe.12614

32. Cabrera D, Wolber FM, Dittmer K, Rogers C, Ridler A, Aberdein D, et al. Glucocorticoids affect bone mineral density and bone remodelling in OVX sheep: A pilot study. Bone Rep. 2018 Nov;9:173-80. https://doi.org/10.1016/j.bonr.2018.11.001

33. Weinstein RS. Glucocorticoids, osteocytes, and skeletal fragility: the role of bone vascularity. Bone. 2010 Mar;46(3):564-70. https://doi.org/10.1016/i.bone.2009.06.030

34. Yamamoto T, Hirano K, Tsutsui H, Sugioka Y, Sueishi K. Corticosteroid enhances the experimental induction of osteonecrosis in rabbits with Shwartzman reaction. Clin Orthop Relat Res. 1995 Jul;\&NA;(316):235-43. https://doi.org/10.1097/00003086-199507000-00033

35. Chiu CT, Chiang WF, Chuang CY, Chang SW. Resolution of oral bisphosphonate and steroid-related osteonecrosis of the jaw-a serial case analysis. J Oral Maxillofac Surg. 2010 May;68(5):1055-63. https://doi.org/10.1016/i.joms.2009.12.030

36. Sousa LH, Moura EV, Queiroz AL, Val D, Chaves H, Lisboa M, et al. Effects of glucocorticoid-induced osteoporosis on bone tissue of rats with experimental periodontitis. Arch Oral Biol. 2017 May;77:55-61. https://doi.org/10.1016/i.archoralbio.2017.01.014 